\title{
Robotic uretero-ureterostomy of the retrocaval ureter without excision of the retrocaval segment
}

\author{
Waleed K. Alkhudair, MD, FRCSC; Raouf Seyam, MD; Hassan M. Al Zahrani, MD, FRCSC; Mohammed F. Al \\ Otaibi, MD, FRCSC; Waleed Al Taweel, MD, FRCSC
}

Department of Urology, King Faisal Specialist Hospital and Research Centre, Riyadh, Kingdom of Saudi Arabia

Cite as: Can Urol Assoc J 2012;6(2):e38-41. hittp://dx.doi.org/10.5489/cuaj.10121

\section{Abstract}

Robotic reconstruction of the retrocaval ureter is gaining momentum as the method of choice for surgically treating this rare condition. Maintaining the retrocaval ureteric segment further facilitates the procedure. We report a case of a 23 -year-old man who underwent intraperitoneal robotic resection anastomosis and repositioning of the retrocaval ureter. We also discuss the advantages of this technique.

\section{Introduction}

Retrocaval ureter is a rare condition which leads to obstruction of the right kidney. The condition becomes symptomatic usually by the third decade of life. ${ }^{1}$ Minimally-invasive surgical management has emerged as the method of choice for repair. Variations in the reported laparoscopic techniques include transperitoneal laparoscopic repair, retroperitoneal laparoscopic repair and single port laparoscopic repair. ${ }^{2-5}$ Few cases of pure robotic repair are reported. ${ }^{6-8}$ The use of the surgical robot facilitates the most challenging part of the procedure, which is intracorporeal suturing. ${ }^{9}$ A surgical variation leading to preservation of the retrocaval segment facilitates the repair. ${ }^{10}$ We report on a case of retrocaval ureter which we treated by pure robotic surgery with preservation of the retrocaval segment.

\section{Case report}

A 23-year-old male patient developed a left renal stone which passed spontaneously. In the course of evaluation for the stone, he was diagnosed as having right retrocaval ureter. Laboratory investigations showed normal urine analysis and renal function. Computerized tomography (CT) showed hydronephrosis and dilatation of the upper third of the right ureter, which curved posterior to the inferior vena cava (IVC) passing to its medial then anterior surface and down to the bladder (Fig. 1). A right retrograde study confirmed the obstruction was caused by the retrocaval position and showed the normal calibre of the retrocaval ureteric segment (Fig. 2). Using the da Vinci Surgical Robotic System (Intuitive Surgical Inc., Sunnyvale, CA), an elective resection anastomosis and repositioning of the ureter was carried out via a transperitoneal approach (Video 1). In the right lateral position, 3 trocars were inserted for the camera and robotic arms. The procedure started by dissecting the right colon. Using blunt and sharp dissection and electrocautary, the renal pelvis was identified. The interaortocaval ureter was dissected (Fig. 3). Transection of the retrocaval segment was carried out and the ureter repositioned lateral to the IVC. Spatulation of the ureteric ends was fashioned. A 5 French double J stent was inserted through the assistant port into the abdominal cavity. The stent was passed using the robotic arms in an antegrade fashion into the distal ureter down to the urinary bladder and in a retrograde fashion into the proximal ureter up to the renal pelvis. Anastomoses of the ureter over the stent was completed with interrupted 4 zero absorbable sutures (Fig. 4). A drain was left in place, the robot and trocars were removed and the abdominal wall defects were closed in layers. The operative duration was 90 minutes, of which 15 minutes were dedicated to ureteric suturing. The estimated blood loss was $100 \mathrm{~mL}$.

Follow-up CT 3 months postoperatively showed a resolution of hydro-ureteronephrosis and the patient remained asymptomatic (Fig. 5).

\section{Discussion}

Our patient had an incidental diagnosis of retrocaval ureter. The younger age of the patient probably contributed to the lack of symptoms, which usually develop in the third or fourth decade of life. The natural course of nonsurgical management of retrocaval ureter is not known in the literature. Hydronephrosis and dilatation of the proximal ureter, 


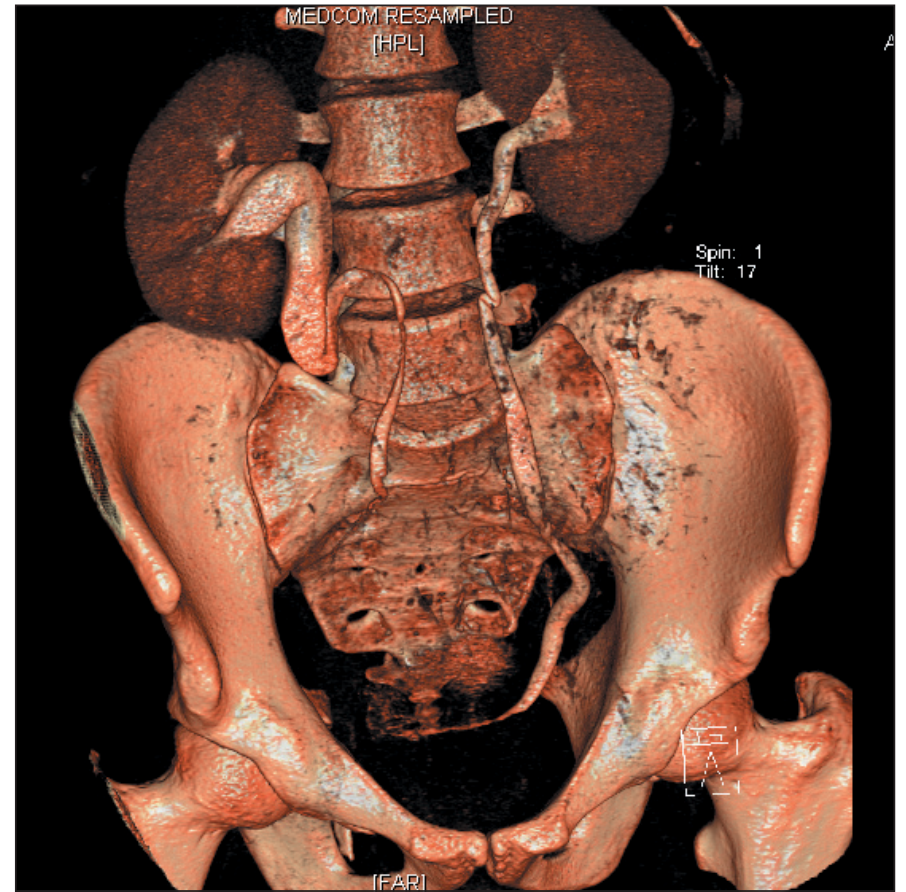

Fig. 1.3D reconstructed computed tomography image showing the course of the right retrocaval ureter.

as confirmed by retrograde study, prompted us for active management. We elected to surgically treat the patient to prevent further dilatation and any deterioration of the renal function.

Case reports of successful laparoscopic dismembered pyeloplasty were described for correction of the retrocaval ureter. $^{2-3}$ The advantages of this technique are minimal blood loss, no reported complications and rapid convalescence. However, the operative time varied from 180 to 560 minutes. The most challenging part of the procedure is intracorporeal suturing. ${ }^{9}$ Excision of the retrocaval ureter segment is usually carried out, but this is controversial. ${ }^{10-12}$ In a limited number of patients, transection of the distal dilated part of the renal pelvis and preservation of the retrocaval segment facilitated the repair and shortened operative time with good outcome. ${ }^{10}$ An intraoperative retrograde peylogram might aid in the decision to preserve the retrocaval segment. ${ }^{3}$ We performed a retrograde study under general anesthesia prior to surgery to confirm the diagnosis of retrocaval ureter and to evaluate the retrocaval ureteric segment for atresia. Alternatively, an intraoperatice retrograde study and attempted ureteric stent insertion would have been carried out. However, we elected to perform robotic surgery at a separate occasion after proper diagnosis and assessment. We preferred not to insert a ureteric stent endoscopically to avoid challenging the kinked ureter. We opted for intraperitoneal insertion of double J stent because it is simple and frequently carried out during robotic pyeloplasty in our centre. Other variations of laparoscopic repair of the retroca-

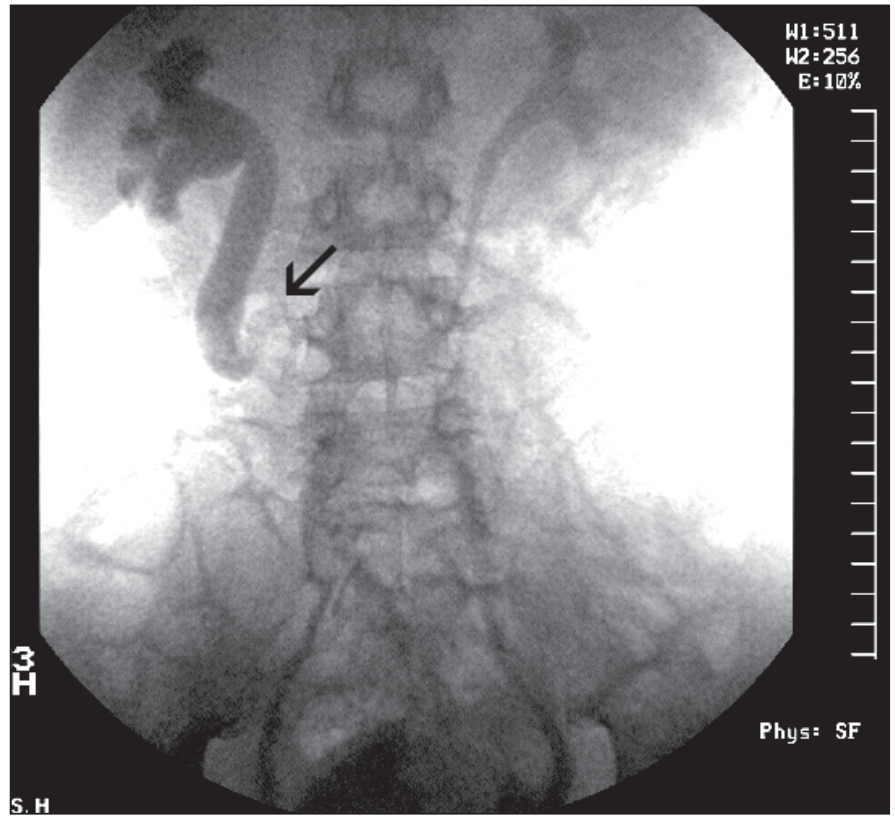

Fig. 2. Right retrograde ureterogram showing a non-atritic retrocaval segment (arrow).

val ureter were reported. Single port laparoscopic repair had comparable result. ${ }^{5}$ Reduction of the number of ports is an advantage that needs to be weighed against the decreased difficulty of the technique, particularly for suture application. Retroperitoneal laparoscopic repair had comparatively good results, shorter operative time (129 minutes, range: 97-189) and minimal blood loss. ${ }^{4}$ However, this technique requires advanced laparoscopic skills, particularly with suturing compared to the transperitoneal approach. ${ }^{13}$ The introduction of robotic-assisted surgery has facilitated intracorporeal suturing during reconstruction. Few cases were reported of pure robotic retrocaval ureter repair. ${ }^{6-8}$ Experienced lapa-

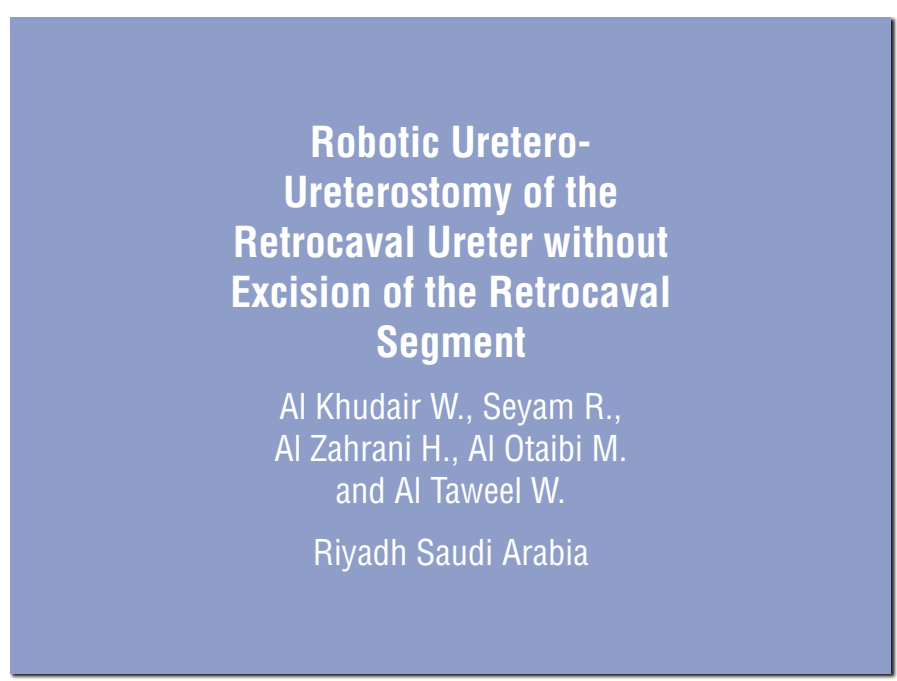

Video 1. Video segment showing the steps of robotic assisted reconstruction of the retrocaval ureter. 


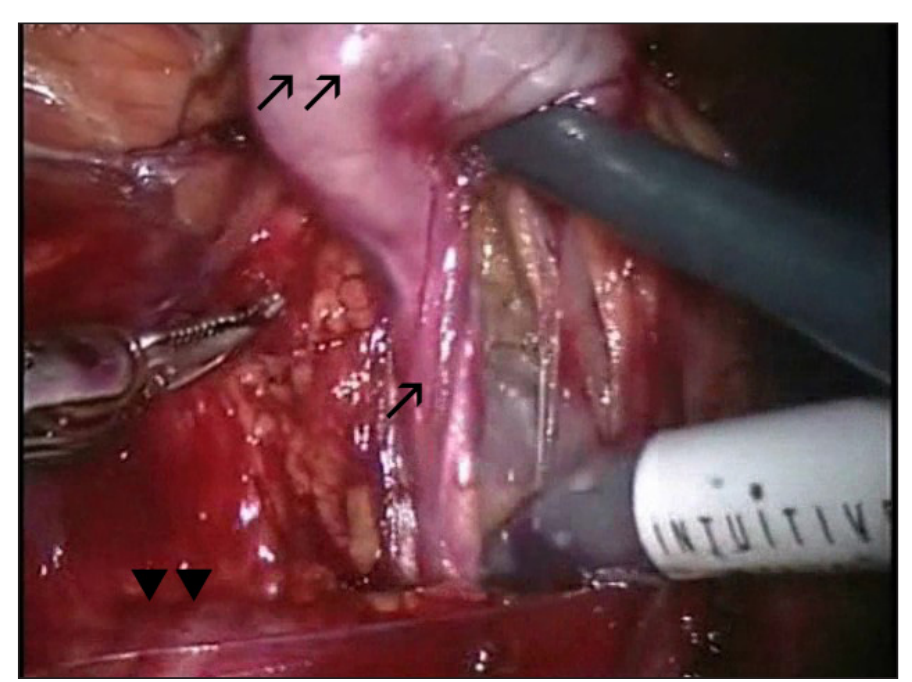

Fig. 3. Intraoperative photograph showing the retrocaval ureteric segment (arrow), dilated renal pelvis (double arrows) and IVC (arrow heads).

roscopists found no significant advantage of robotic over laparoscopic reconstruction. ${ }^{7}$ The operative duration and

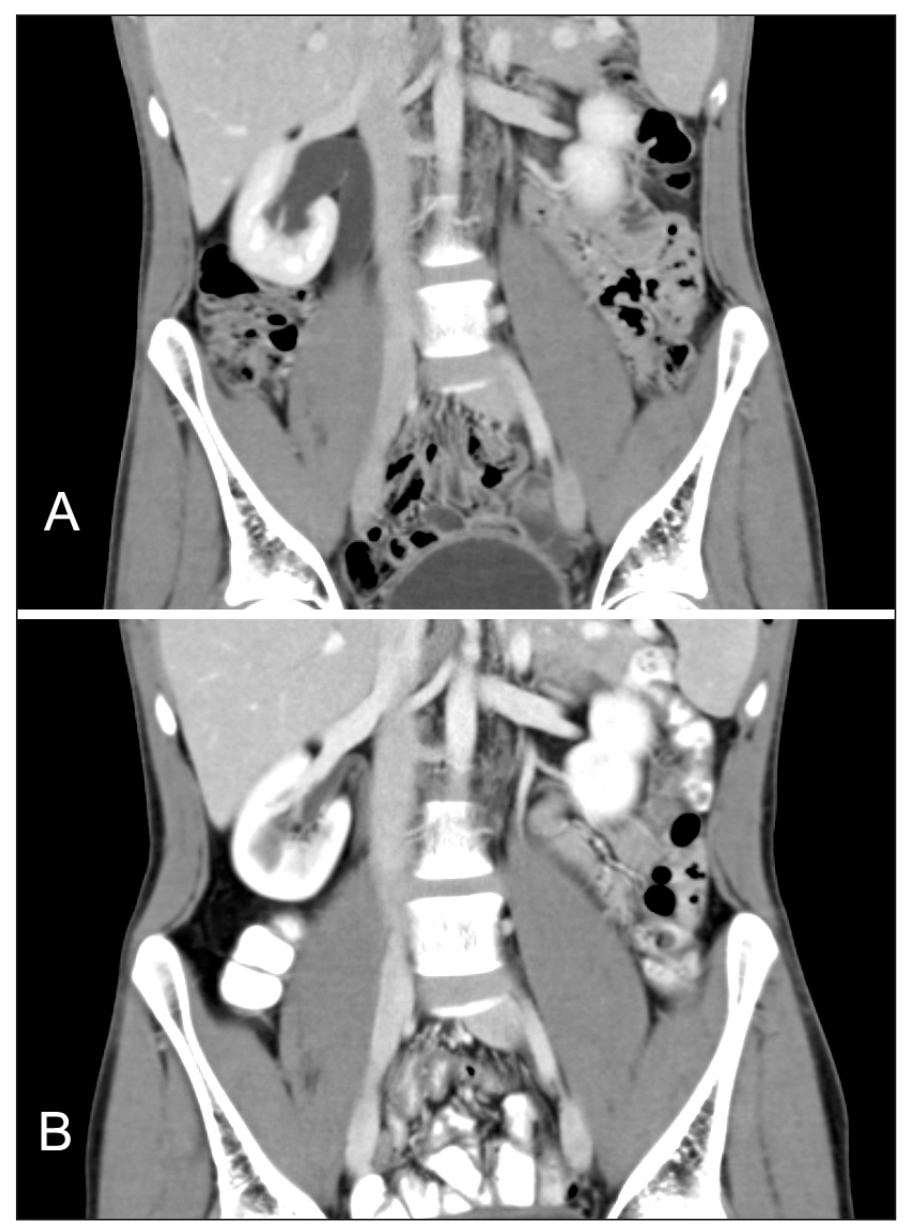

Fig. 5. Coronal reconstruction of computed tomography scan of the abdomen during the nephrogram phase showing $A$. dilated right renal pelvis before surgery and B. 3 months post surgery with disappearance of dilatation.

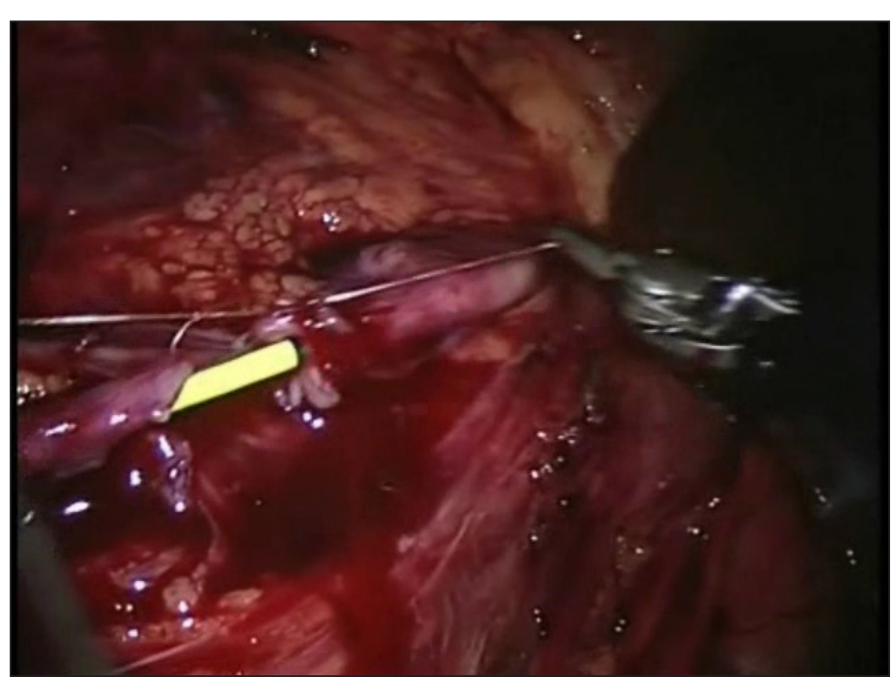

Fig. 4. Intraoperative photograph showing both ends of transected ureter with placement of the first anastomotic suture over a double $\mathrm{J}$ stent.

blood loss in our case are comparable to those reported by others. The average time for robotic retrocaval ureter reconstruction was 80 minutes (range: 75-90) and the average blood loss was $100 \mathrm{~mL} .^{8}$ Only long-term comparative studies can show the advantages of each technique. With the rarity of cases and sporadic reporting of different techniques, we feel that robotic reconstruction of the retrocaval ureter and intraperitonieal ureteric stent insertion are simple and have an excellent uneventful outcome. Preservation of the retrocaval segment, in selected cases where there is no evidence of atresia on imaging, further facilitates the technique.

\section{Conclusion}

Robotic surgery and preservation of the retrocaval segment facilitate the reconstruction of the retrocaval ureter.

Competing interests: None declared.

This paper has been peer-reviewed.

\section{References}

1. Yarmohammadi A, Mohamadzadeh Rezaei M, Feizzadeh B, et al. Retrocaval ureter: a study of 13 cases. Urol J 2006:3:175-9.

2. Chung BI, Gill IS. Laparoscopic dismembered pyeloplasty of a retrocaval ureter: case report and review of the literature. Eur Urol 2008;54:1433-6. hittp://dx.doi.org/10.1016/i.eururo.2008.09.010

3. Dogan HS, Oktay B, Vuruskan H, et al. Treatment of Retrocaval Ureter by Pure Laparoscopic Pyelopyelostomy: Experience on 4 Patients. Urology 2010;75:1343-7. hittp://dx.doi.org/10.1016/i. urology.2009.09.040

4. $\mathrm{XU}_{\mathrm{U}} \mathrm{DF}, \mathrm{Y}_{a 0} \mathrm{YC}$, Ren IZ, et al. Retroperitoneal laparoscopic ureteroureterostomy for retrocaval ureter: report of 7 cases. Urology 2009;74:1242-5. nitp://dx.doi.org/10.1016/i.urology.2009.04.097

5. Autorino R, Khanna R, White MA, et al. Laparoendoscopic Single-site Repair of Retrocaval Ureter: First Case Report. Urology 2010 Apr 7. [Epub ahead of print]

6. Gundeti MS, Duffy PG, Mushtaq I. Robotic-assisted laparoscopic correction of pediatric retrocaval ureter. I Laparoendosc Adv Surg Tech A 2006; 16:422-4. Ditp://dx.doi.org/10.1089//ap.2006.16.422 
Robotic uretero-ureterostomy of the retrocaval ureter

7. Hemal AK, Rao R, Sharma S, et al. Pure robotic retrocaval ureter repair. Int Braz I Urol 2008;34:734-8. hitp://dx.doi.org/10.1590/S1677-55382008000600008

8. Hemal AK, Nayyar R, Gupta NP, et al. Experience With Robot Assisted Laparoscopic Surgery for Upper and Lower Benign and Malignant Ureteral Pathologies. Urology 2010 Mar 27. [Epub ahead of print] PubMed PMID: 20350753

9. Baba $S$, Oya $M$, Miyahara $M$, et al. Laparoscopic surgical correction of circumcaval ureter. Urology 1994;44:122-6. hitr://dx.doi.org/10.1016/S0090-4295(94)80023-5

10. Simforoosh N, Nouri-Mahdavi K, Tabibi A. Laparoscopic pyelopyelostomy for retrocaval ureter without excision of the retrocaval segment: first report of 6 cases. J Urol 2006; 175:2166-9; discussion 2169. hittp://dx.doi.org/10.1016/S0022-5347(06)00269-2

11. Bagheri F, Puszzai C, Szántó A, et al. Laparoscopic repair of circumcaval ureter: one-year follow-up of three patients and literature review. Urology 2009;74:148-53. Hitp://dx.doi.org/10.1016/i.urol ggy.2009.02.048
12. Zhang XD, Hou SK, Zhu JH, et al. Diagnosis and treatment of retrocaval ureter. Eur Urol 1990; 18:207-10.

13. Ramalingam M, Selvarajan K. Laparoscopic transperitoneal repair of retrocaval ureter: report of two cases. J Endourol 2003;17:85-7. hitp://dx.doi.org/10.1089/08927790360587397

Correspondence: Dr. Waleed K. Alkhudair, Chairman Department of Urology, King Faisal Specialist Hospital and Research Centre, Riyadh, Saudi Arabia and Professor, College of Medicine, Al Faisal University, Riyadh, Saudi Arabia, King Faisal Specialist Hospital \& Research Center, P.0. 3354 (MBC83), Riyadh, Saudi Arabia 11211; fax: +966-01-442-4301; walkhudair@gmail.com 